\title{
EchoGéo
}

36 | 2016

Stratégies de villes et "modèles urbains"

\section{Patrimoines et territoires : des objets communs aux archéologues et géographes}

\section{Laurence Gillot}

\section{(2) OpenEdition \\ 1 Journals}

\section{Electronic version}

URL: https://journals.openedition.org/echogeo/14612

DOI: 10.4000/echogeo.14612

ISSN: 1963-1197

\section{Publisher}

Pôle de recherche pour l'organisation et la diffusion de l'information géographique (CNRS UMR 8586)

\section{Electronic reference}

Laurence Gillot, "Patrimoines et territoires : des objets communs aux archéologues et géographes", EchoGéo [Online], 36 | 2016, Online since 30 June 2016, connection on 10 August 2021. URL: http:// journals.openedition.org/echogeo/14612 ; DOl: https://doi.org/10.4000/echogeo.14612

This text was automatically generated on 10 August 2021

EchoGéo est mis à disposition selon les termes de la licence Creative Commons Attribution - Pas d'Utilisation Commerciale - Pas de Modification 4.0 International (CC BY-NC-ND) 


\title{
Patrimoines et territoires : des objets communs aux archéologues et géographes
}

\author{
Laurence Gillot
}

1 Patrimoine et territoire constituent deux objets communs, autant dans le sens de « partagés » que de «courants », aux archéologues et géographes. Ces deux termes ont connu au cours des deux dernières décennies une inflation importante, que l'on peut mettre globalement en lien avec le tournant post-moderne (Poulot, 2000, 2006) et postcolonial (Turgeon, 2003). De fait, ces deux objets, d'un côté conceptuels et académiques, et de l'autre politiques et socio-culturels, ont permis de nouvelles avancées dans le dialogue entre géographes et archéologues, en même temps que ces échanges ont permis de renouveler leur étude, d'une part, et leur gestion dans le présent, d'autre part (Demoule et Stiegler, 2008; Djament-Tran, 2015; Gravari-Barbas et GuichardAnguis, 2003; Veschambre, 2007). C'est cet aspect de l'archéogéographie, comme articulation des savoirs scientifiques et endogènes, mais aussi comme co-construction de problématiques et de méthodes communes, que nous proposons de traiter ici, à travers l'exemple d'un programme de recherche interdisciplinaire mené dans l'oasis de Figuig au Maroc (Gillot et Vallat, 2014).

2 Dans le cadre d'une convention avec l'Institut National des Sciences de l'archéologie et du patrimoine (INSAP) du Maroc et avec la Municipalité de Figuig, une équipe de chercheurs français, actuellement dirigée par André Del (École d'architecture Paris Valde-Seine, EVCAU) et moi-même, mène des actions d'inventaire, de recherche et de valorisation des patrimoines de l'oasis de Figuig depuis 2006. Figuig est une oasis marocaine de la région de l'Oriental au Maroc, située sur la frontière avec l'Algérie, à environ $400 \mathrm{~km}$ au sud de la Méditerranée et à $7 \mathrm{~km}$ de la ville algérienne de Beni Ounif. Sa situation stratégique aux portes du désert permit à Figuig de jouer, tout au long de son histoire, le rôle de relais sur les axes du commerce caravanier transsaharien et sur l'axe sub-saharien est-ouest. Mais la fermeture de la frontière entre les deux pays a engendré l'isolement et l'appauvrissement de l'oasis, déjà touché par une crise 
économique et un exode démographique depuis les années 1970. Dans ce contexte, l'oasis connait des mutations socio-spatiales importantes se traduisant par la dégradation des architectures et le mitage des tissus urbains dits traditionnels (et $a$ contrario l'expansion de nouvelles constructions sur des modèles exogènes), l'abandon des jardins (et l'introduction de pratiques nouvelles conduisant à la surexploitation de certaines parcelles) et la disparition de certaines pratiques sociales dites ancestrales (avec notamment l'importation de nouvelles pratiques par les membres de la diaspora). Pour les acteurs locaux, comme pour les acteurs de la coopération, l'oasis doit donc faire face à un processus d'érosion des patrimoines, rendant de plus en plus urgente la prise de mesures de sauvegarde. Dans ce contexte, le collectif, associant géographes, historiens, archéologues et architectes fut notamment chargé, à la demande de la municipalité, de coordonner les études documentaires pour le dossier d'inscription de Figuig au patrimoine mondial de l'Unesco, permettant que l'oasis figure sur la liste indicative en juin 2011. Les chercheurs mènent aussi des fouilles et prospections archéologiques, des relevés architecturaux et topographiques, et des études environnementales avec deux objectifs corrélés : celui d'une étude des dynamiques de peuplement et celui d'un projet de réhabilitation et de sauvegarde des patrimoines figuiguiens ${ }^{1}$.

3 L'équipe s'est donc donné pour objectif d'analyser cet espace en croisant des échelles et des temporalités multiples, le tout dans un cadre territorial qui n'a cessé d'évoluer et où se juxtaposent des héritages et des conceptions autochtones, tribales de l'espace et des conceptions modernes, importées par l'administration française sous le protectorat, et, plus récemment, par les acteurs de la coopération (Conseil régional de Seine Saint-Denis, Mon-3, Africa 70). Il s'agit donc de travailler sur l'évolution de cet espace selon une approche dynamique et multiscalaire, tenant compte de la manière dont les diverses populations, berbères, arabes, chrétiennes, juives, ont investi l'espace, l'ont modelé et remodelé. Cela passe notamment par une analyse des termes utilisés pour nommer ce territoire : oasis, ville, palmeraie, ksour, etc. sont ainsi considérés comme l'expression de territorialités multiples. De fait, les concepts de «transformission » et de "résilience », issus de l'archéogéographie, trouvent tout leur intérêt dans cette recherche qui part du présent et des représentations sociales de l'espace pour comprendre la dynamique de constitution de ce territoire. L'idée est aussi que l'étude des formes paysagères, urbaines et architecturales sur la longue durée, et la documentation des usages passés et présents, pourraient être potentiellement instructifs pour les populations locales et les autorités, qui sont confrontées aujourd'hui à des défis importants concernant l'usage des ressources et la sauvegarde des patrimoines. De fait, notre démarche vise aussi à comprendre ce qui fait patrimoine pour les uns et les autres, ce qu'il importe de recevoir et de transmettre, en tenant compte de la fonction sociale de ces héritages et de leur place dans la structuration de l'espace. En d'autres termes, nos recherches portent sur les processus de territorialisation et de patrimonialisation, et sur leur articulation ${ }^{2}$. Loin d'adopter une définition préconçue du patrimoine et du territoire, nous cherchons à articuler les conceptions institutionnelles, savantes et sociales (Gillot, Del et Cohen, 2012). Notre démarche se fonde donc aussi sur une approche participative qui associe les acteurs locaux, individuellement et collectivement, dans une forme de "recherche-action ». Ainsi, nous pensons éviter d'apporter des solutions exogènes déconnectées et uniquement inspirées de notre propre perception du patrimoine et du territoire. 
4 Pour répondre à ces objectifs, nous avons opté pour une approche interdisciplinaire en associant les méthodes, outils et concepts des archéologues, historiens, géographes et anthropologues. Les fouilles archéologiques du ksar des Ouled Jaber en sont un bon exemple. Sur la base d'un travail de prospection réalisé en 2009-2010 par Jean-Pierre Vallat, Claire Pichard et Ricardo Villaescusa, en particulier le long du rempart et sur les ruines d'une ancienne mosquée, nous nous sommes fixé pour objectif d'éclairer l'histoire du ksar des Ouled Jaber, et plus largement l'histoire ancienne de Figuig aux époques médiévale et moderne, pour lesquelles les sources historiques sont très lacunaires et disparates (Gillot, 2014). Le ksar abandonné des "Ouled Jaber" (descendants de Jaber), ainsi dénommé en référence à l'une des communautés qui l'a habité, vraisemblablement, entre le XIII et le XVIII ${ }^{e}$ siècle, se trouve sur le plateau de Figuig, aux confins des ksour de Loudaghir et Laabidat ${ }^{3}$. Le ksar des Ouled Jaber formait un quartier résidentiel doté, comme les autres ksour, d'une enceinte, de tours et de portes d'accès, ainsi que d'habitations regroupées en quartiers qui rassemblaient les familles d'un même lignage. Il est actuellement laissé à l'abandon et la majeure partie de ses structures sont enfouies ou dégradées. Les fouilles archéologiques visent ainsi à préciser les conditions d'implantation du ksar et l'histoire des origines de la tribu des Jaber, la nature des structures qu'il contient (édifices religieux, habitats, bains, etc.), son fonctionnement et sa relation avec les autres ksour (coexistence, conflits, etc.) avant de comprendre les conditions de son abandon et de sa destruction, phases sur lesquelles quelques sources écrites et orales nous renseignent ${ }^{4}$. Il s'agit aussi d'appréhender le rapport entre les formes spatiales et les structures sociales, de même que les liens entre l'espace bâti, les sources d'eau et la palmeraie voisine ${ }^{5}$. Le programme s'inscrit enfin dans une perspective patrimoniale, visant à atténuer les conflits mémoriels et à permettre une appropriation de ce site par l'ensemble de la population figuiguienne.

5 Les prospections archéologiques et les fouilles ont été étroitement associées à l'étude urbaine et architecturale menée par les architectes sur les espaces « urbanisés » et les architectures (de terre) en interrogeant l'évolution des formes urbaines et architecturales sur la longue durée. Nous postulons que la société oasienne est loin d'être figée et que l'espace est sans cesse recomposé, tant pour répondre à des besoins évolutifs qu'en raison des conflits d'appropriation entre les communautés de l'oasis. Le temps long souligne en outre l'arrivée mais aussi le départ de certaines communautés qui ont plus ou moins marqué l'espace et dont l'héritage a été traité de manière variable par les populations successives ${ }^{6}$. Parallèlement, nous travaillons sur les généalogies familiales et la mémoire orale des partages de l'espace qui ne donnent lieu à aucune consignation, dans un cadastre notamment. Ce travail d'enquête auprès des populations et différents clans vise autant à comprendre la structuration de l'espace que les enjeux mémoriels pour sa réutilisation et/ou son abandon. L'enquête ethnographique et anthropologique, basée sur l'observation des pratiques constructives, nous permet également de travailler sur l'évolution des typologies et fonctions des architectures.

6 Le ksar des Ouled Jaber représente ainsi un terrain d'étude où il nous semble possible d'observer ces dynamiques sur la longue durée, et de mettre en lumière autant les continuités que les discontinuités dans l'aménagement de cet espace. De fait nous avons $\mathrm{pu}$ distinguer plusieurs formes d'appropriation/désappropriation de cet espace «abandonné » qu'est le ksar des Ouled Jaber: espace mémoriel, espace-vide (no man's 
land), espace dénigré (utilisé comme décharge), lieu de passage. Au final, ces connaissances permettent de comprendre les enjeux de la (non) sauvegarde de cet espace. Cela ouvre de fait des perspectives lorsque l'on envisage la possibilité de faire de ce ksar un lieu commun aux différentes communautés, celui d'une histoire partagée, support de mémoires tant individuelles que collectives. Or, c'est par le croisement des regards des différentes disciplines associées à ce programme de recherche que ces perspectives sont possibles.

7 Sur le plan de la méthode, la principale difficulté résidait dans la grande diversité et disparité des sources relatives à l'histoire de Figuig. Cela nous a précisément engagé à mener un travail interdisciplinaire et à réfléchir constamment à la manière de récolter, consigner, traiter, recouper et interpréter nos sources. Nous avons ainsi mobilisé des outils communs à la géographie en rassemblant des cartes anciennes, vues aériennes et observations de terrain en vue de proposer une interprétation des vestiges et d'orienter nos fouilles. Le recoupement avec le témoignage d'un géographe français ${ }^{7}$ a également permis de construire un certain nombre d'hypothèses.

8 Enfin, un autre point de rapprochement avec la géographie est la mise en place d'un système d'information géographique (SIG) archéologique et patrimonial permettant de gérer et interpréter l'ensemble des données récoltées et produites (Gillot et Del, 2012). Dans ce contexte, nous avons réalisé une carte du « potentiel » archéologique. L'objectif est de constituer une base de données pour le développement futur de fouilles archéologiques et de recherches historiques permettant d'éclairer les dynamiques territoriales propres à l'Afrique du Nord depuis l'Antiquité. Nous avons jusqu'à présent sondé l'oasis et sa région au cours de deux missions, en recoupant les cartes anciennes dont nous disposons, les vues aériennes, les informations orales et nos observations sur le terrain. Ces premières prospections révèlent la richesse archéologique de l'oasis et de sa région, et devra s'accompagner d'un important travail de qualification de ces lieux, déjà entrepris dans le cadre du labex Dynamite ${ }^{8}$. Enfin, nous estimons qu'un tel SIG permettrait de mettre cette documentation en perspective avec des actions concrètes visant la sauvegarde et la valorisation du patrimoine, à travers la mise en place d'une plateforme numérique de partage où des non-experts pourraient également partager leur «savoir ». Cette approche, fondée sur l'idée que le partage des savoirs permettrait une appropriation par l'ensemble des acteurs et donc une meilleure sauvegarde des patrimoines, s'intègre dans un ensemble d'expériences similaires menées au niveau international, notamment la plateforme historypin (A global community collaborating around history), prenant part à ce que les initiateurs de ces projets appellent des " projets collaboratifs d'indexation sociale »'. Au terme du projet, le SIG sera mis en ligne et les données scientifiques qu'il contient seront exploitables tant par la communauté scientifique que par chaque utilisateur du web.

En conclusion, cette présentation succincte du programme mené dans l'oasis de Figuig montre que les recherches sur les patrimoines et les territoires ont tout à gagner du dialogue renouvelé entre archéologues et géographes. Ce projet montre également le développement d'une nouvelle forme d'interdisciplinarité où tous les chercheurs intéressés par le "social » réfléchissent ensemble et co-construisent des concepts et des outils à la manière des social, heritage ou cultural studies anglo-saxonnes. Ce décloisonnement participe aussi d'une réflexivité accrue dans nos disciplines respectives et d'un rapprochement entre les mondes professionnels et académiques, les mondes savants et non experts. C'est là tout l'enjeu actuel du métier 
d'archéogéographe, c'est-à-dire produire un savoir partagé qui lui-même puisse guider le développement futur des sociétés.

\section{BIBLIOGRAPHY}

Demoule J.-P., Stiegler B. (éds.), 2008. L'avenir du passé: Modernité de l'archéologie. I.N.R.A.P., La Découverte, Paris.

Di Méo G., 1994. Patrimoine et territoire, une parenté conceptuelle. Espaces et sociétés, 78, p. 15-34.

Djament-Tran G., 2015. La (dé)territorialisation et les changements d'échelle du patrimoine. Espacestemps.net, 20/06/2015, p. 1-22.

Gautier E. F., 1917. La source de Tzaddert à Figuig. Annales de Géographie, 26 (144), p. 453-466.

Gillot L., 2014. Le ksar abandonné des Ouled Jaber. In Vallat J.-P., (éd.), Le patrimoine marocain : Figuig, une oasis au cœur des cultures. Paris, L'Harmattan, p. 243-284

Gillot L., Del A., 2012. Preparation and Submission of the nomination File of the Oasis of Figuig (Morocco) for inscription on the WHL: impacts and uses of a GIS. Geoinformatics, 6, p. 140-149.

Gillot L., Del A. et Cohen M., 2012. Patrimoine et développement local : pratiques institutionnelles et pratiques sociales. In Actes du symposium scientifique de la 17ème Assemblée générale de l'ICOMOS, Paris.

Gillot L., Vallat J.-P., 2014. Les enjeux de la sauvegarde des patrimoines de l'oasis de Figuig (Maroc) »/ The territorial Stakes of the Safeguard of the Heritage in the Oasis of Figuig (Morocco). In Carcaud N. et Arnaud-Fassetta G., La géoarchéologie au XXIème siècle. French Geoarchaeology in the 21st century. CNRS Éditions, Paris, p. 195-202/193-200.

Gravari-Barbas M., Guichard-Anguis S. (éds.), 2003. Regards croisés sur le patrimoine dans le monde à l'aube du XXIe siècle. Paris, Presses de l'Université de Paris-Sorbonne.

Janty G., 2014. Les enjeux de la préservation et du développement d'un paysage culturel. Le cas de la palmeraie de l'oasis de Figuig (Maroc). Thèse de doctorat en géographie sous la direction de $\mathrm{M}$. Cohen, Université Paris 7-Denid Diderot.

Poulot D., 2000. Patrimoine et modernité. Paris, L'Harmattan.

Poulot D., 2006. De la raison patrimoniale aux mondes du patrimoine. Socio-Anthropologie, 19, mis en ligne le 31 octobre 2007. URL : http://socio-anthropologie.revues.org/753

Turgeon L., 2003. Patrimoines métissés. Contextes coloniaux et postcoloniaux. Paris, Maison des Sciences de l'Homme.

Vallat J.-P., 2014, (éd.), Le patrimoine marocain : Figuig, une oasis au cour des cultures, L'Harmattan, Paris.

Veschambre V., 2007. Patrimoine : un objet révélateur des évolutions de la géographie et de sa place dans les sciences sociales. Annales de géographie, 4, p. 361-381. 


\section{NOTES}

1. Plus largement, ces recherches s'inscrivent dans un programme visant à développer les connaissances de l'histoire du peuplement de l'oasis et de la région de l'Oriental. La présence de traces archéologiques remontant à la préhistoire (peintures rupestres notamment) et l'inscription dans l'espace du passage et de la présence de communautés berbères, juives, chrétiennes et musulmanes indiquent en effet l'importance historique de l'oasis et de sa région. Voir Vallat J.-P., 2014.

2. Nous nous inspirons ici des travaux de Guy Di Méo, qui a très bien souligné la parenté conceptuelle entre les deux notions de patrimoine et territoire (Di Méo, 1994).

3. Actuellement, Figuig est constituée d'un noyau urbain de 7 ksour (Laâbidate, Lamaïz, Hammam Foukani, Hammam Tahtani, Loudaghir, Ouled Slimane et Zenaga) et de quartiers plus récents. Ces ksour sont reliés entre eux par l'extension récente de l'espace bâti ou par la palmeraie qui s'étend sur environ 650 ha.

4. La fouille entreprise depuis 2010 s'est plus particulièrement concentrée au niveau de la "mosquée », située au sud-est du ksar. Il ne reste à présent que trois arcs en place, dont un, au nord, a été restauré en 2000. Si l'identification à une mosquée semble assurée par le recoupement de nos sources, l'agencement des vestiges demeure peu évident, et il est à ce jour encore impossible d'établir une chronologie exacte des fondations, des niveaux d'occupation, d'abandon et de destruction de la mosquée et du ksar. Nous avons essentiellement fouillé des couches d'abandon après destruction, dans une zone qui a elle-même été fortement perturbée par des travaux divers : creusement du fossé dans la seconde moitié du XIX ${ }^{\mathrm{e}}$ siècle, creusement du puits au milieu du $\mathrm{XX}^{\mathrm{e}}$ siècle, restauration de l'arc nord et construction du mur de retenue au sud (années 1990 et 2000).

5. L'évolution des espaces de jardins de la palmeraie a été étudiée par G. Janty, qui a pu montrer, sur la base de vues aériennes anciennes et récentes et d'observations de terrain, les trajectoires variées de la palmeraie, entre régression, reprise et stagnation, mettant ainsi à l'épreuve un discours dénonçant la dégradation générale du patrimoine naturel de l'oasis (Janty, 2014).

6. On peut citer à cet égard l'abandon d'un cimetière chrétien ou la reconversion de l'unique église en centre du patrimoine, ou encore l'état de dégradation alarmant du mellah, quartier juif abandonné du ksar Loudaghir, malgré des fouilles de sauvetage entre 2005 et 2009. Si ces espaces ne sont pas détruits, réappropriés ou reconstruits, l'on doit y voir le signe de conflits persistants sur leur usage entre les différents acteurs en présence.

7. Le texte de Émile-Félix Gauthier (1864- ?), professeur de l'université d'Alger, rapporte des informations orales relatives à l'histoire de Figuig et aux conflits liés à l'appropriation des ressources en eau de la palmeraie. Il offre notamment quelques indications sur les conditions d'abandon du ksar des « Djouaber » et l'état des ruines en 1914 (Gautier, 1917).

8. Adrian Robu, post-doctorant à ANHIMA (UMR 8210), a effectué un post-doctorat financé par le labex Dynamite (Dynamiques territoriales) dans le GT « Les systèmes de peuplement sur le temps long: temporalités et spatialités, réseaux et territoires » pour deux projets (CIRCE et Figuig) mobilisant les savoirs géographiques et archéologiques dans une approche interdisciplinaire qui s'intègre elle-même dans un projet plus large d'« humanités numériques ».

9. Il s'agit donc de développer une nouvelle pratique collaborative de recherche qui vise à élaborer des méthodes et des dispositifs permettant à chaque groupe d'informations de valider l'autre, au lieu de fusionner les informations "universitaires" et "citoyennes". Dans ce cadre, les informations "universitaires" sont amenées à qualifier les informations déposées par "les citoyens". Ces informations ainsi qualifiées sont alors validées, ce qui permet l'utilisation des informations " citoyennes" comme source d'informations pertinentes. 


\section{AUTHOR}

\section{LAURENCE GILLOT}

Laurence Gillot, Laurence.Gillot@ulb.ac.be, est Maître de conférences à l'Université Paris Diderot et membre de l'UMR 8210 ANHIMA (Anthropologie et Histoire des Mondes Anciens). 